\title{
IKBKB expression in clear cell renal cell carcinoma is associated with tumor grade and patient outcomes
}

\author{
BARTLOMIEJ E. KRAZINSKI ${ }^{1}$, ANNA E. KOWALCZYK ${ }^{1}$, AGNIESZKA SLIWINSKA-JEWSIEWICKA ${ }^{1}$, \\ JEDRZEJ GRZEGRZOLKA ${ }^{2}$, JANUSZ GODLEWSKI ${ }^{1}$, PRZEMYSLAW KWIATKOWSKI ${ }^{1}$, \\ PIOTR DZIEGIEL $^{2,3}$, ZBIGNIEW KMIEC $^{1,4}$ and JOLANTA KIEWISZ ${ }^{1}$
}

\author{
${ }^{1}$ Department of Human Histology and Embryology, University of Warmia and Mazury in Olsztyn, 10-082 Olsztyn; \\ ${ }^{2}$ Department of Human Morphology and Embryology, Division of Histology and Embryology, Wroclaw Medical University, \\ 50-368 Wroclaw; ${ }^{3}$ Department of Physiotherapy, Wroclaw University School of Physical Education, 51-612 Wroclaw; \\ ${ }^{4}$ Department of Histology, Medical University of Gdansk, 80-211 Gdansk, Poland
}

Received June 21, 2018; Accepted October 24, 2018

DOI: 10.3892/or.2018.6872

\begin{abstract}
Inhibitor of nuclear factor kappa B kinase subunit B (IKBKB or IKK $\beta$ ) is a key activator of the nuclear factor $\kappa \mathrm{B}$ transcription factor pathway. Increased expression and/or aberrant activity of IKBKB have been observed in various types of human cancer. Three independent techniques, reverse transcription-quantitative polymerase chain reaction, western blotting and immunohistochemistry, were used to demonstrate that $I K B K B$ expression is decreased in clear cell renal cell carcinoma (ccRCC). Notably, the patients with upregulated IKBKB protein expression were characterized by higher nuclear grade tumors and significantly shorter survival. The findings indicate that IKBKB protein may be of clinical relevance in ccRCC, serving as a marker of poor prognosis and as potential target for adjuvant chemotherapies. Further studies are required to validate the prognostic and predictive value of IKBKB.
\end{abstract}

\section{Introduction}

Renal cell carcinomas (RCCs) are a heterogeneous group of different kidney tumors, with clear cell RCC (ccRCC) being the most prevalent histological subtype $(1,2)$. The majority of sporadic ccRCC cases are characterized by loss or inactivation of von Hippel-Lindau ( $V H L)$ tumor-suppressor gene, resulting in accumulation of hypoxia inducible factors (HIFs) and overexpression of HIF-driven genes that are partially responsible for cell proliferation, angiogenesis and tumor growth $(1,2)$. If diagnosed at early stages, clear cell tumors are usually curable by surgical treatment; whereas advanced ccRCC are

Correspondence to: Dr Jolanta Kiewisz, Department of Human Histology and Embryology, University of Warmia and Mazury in Olsztyn, 30 Warszawska Street, 10-082 Olsztyn, Poland

E-mail: jolanta.kiewisz@uwm.edu.pl

Key words: inhibitor of nuclear factor- $\mathrm{\kappa B}$ kinase subunit $\mathrm{B}$, expression, clear cell renal cell carcinoma, prognosis, survival characterized by worse prognosis compared to non-clear cell subtypes of RCC (3). The majority patients usually report no symptoms and approximately a third of patients with ccRCC are diagnosed at the metastatic stage of the disease. Despite surgical treatment of primary disease, approximately half of the patients with primarily localized tumors will eventually develop metastases or recurrent disease $(1,3)$. The molecular background of ccRCC has been extensively studied resulting in better understanding of the ccRCC molecular background and development of novel therapeutic strategies $(2,4)$. However, the benefits of adjuvant treatments for advanced or metastatic ccRCC still require improvement (1). In addition, there is a lack of reliable molecular prognostic and predictive markers that could be routinely used for improved patient monitoring and targeted as effective treatment strategies $(1,5)$.

Nuclear factor $-\kappa \mathrm{B}(\mathrm{NF}-\kappa \mathrm{B})$ is a protein complex that controls the expression of genes involved in immune response and cell survival and is often upregulated in human cancer $(6,7)$ including RCC (8). The classical (canonical) $\mathrm{NF}-\kappa \mathrm{B}$ pathway comprises heterodimer of the transcription factors RelA/p50. In the resting state, heterodimers are sequestered in the cytoplasm by NF- $\kappa \mathrm{B}$ inhibitors (I $\kappa-\mathrm{B})$. Canonical NF- $\mathrm{NB}$ signaling can be induced by pro-inflammatory mediators including lipopolysaccharides, cytokines or CD40 ligand (6,7). Inhibitor of nuclear factor $\kappa \mathrm{B}$ kinase subunit $\mathrm{B}$ (IKBKB or IKK $\beta$ ) is part of the I $\kappa-B$ kinase (IKK) complex that activates the transcription factor NF- $\kappa \mathrm{B}$ (9). Upon activation, IKBKB phosphorylates I $\kappa-\mathrm{B}$ leading to its ubiquitination and degradation, releasing RelA/p50 from inhibition. Following translocation to the nucleus, RelA/p50 binds to $\kappa \mathrm{B}$ sites within promoters and regulates the transcription of target genes to increase the expression of pro-survival and pro-inflammatory factors $(6,7,9)$. Generation of $I \mathrm{KBKB}^{-/-}$mice and further experimentation performed using IKBKB-deficient cells demonstrated that this protein is essential for activation of $\mathrm{NF}-\kappa \mathrm{B}$ and functions as a dominant kinase in the canonical NF- $\mathrm{NB}$ cascade $(6,10,11)$.

Sustained activation, defective regulation and overexpression of proteins of the NF- $\mathrm{B}$ pathway is observed in certain tumors and tumor-derived cell lines, and is associated with the malignant phenotype in the majority of cases (12). NF- $\kappa \mathrm{B}$ 
transcription factors have been extensively researched because of their involvement in stromal communication with cancer cells and role in establishing the tumor microenvironment. Cancer cells, a variety of non-cancerous tumor-associated immune cells and fibroblasts exhibit altered $\mathrm{NF}-\kappa \mathrm{B}$ signaling, which is often associated with intratumoral immunosuppression and development of multidrug resistance $(7,12)$. In addition to its major role in the activation of $\mathrm{NF}-\kappa \mathrm{B}$ pathway, IKBKB was demonstrated to phosphorylate NF- $\kappa \mathrm{B}$-unrelated factors, including tumor suppressor p53 or forkhead box O3 transcription factors, targeting them for degradation by the ubiquitin-proteasome pathway $(13,14)$. It was also demonstrated that IKBKB is a target for prolyl hydroxylase-mediated hydroxylation and, therefore, hypoxia increases the expression and activity of IKBKB in cultured cancer cells (15); whereas the protein product of $V H L$ tumor suppressor gene (pVHL) negatively regulates IKBKB (16).

In the present study, the expression of IKBKB in tumors and matched non-cancerous renal tissues of patients with ccRCC was investigated. The association of IKBKB protein expression with clinicopathological parameters and survival of ccRCC patients was assessed.

\section{Patients and methods}

Patients and the collection of samples. Specimens were obtained from postoperative material of 66 patients with histologically confirmed ccRCC (33 men and 33 women; mean age \pm standard deviation, 63.2 \pm 10.6 ; range $27-83$ years) operated on at the Department of Oncological Surgery, Warmia and Mazury Oncological Center in Olsztyn (Olsztyn, Poland) between March 2010 and July 2014. None of patients had a second neoplastic disease or had previously undergone chemoor radiotherapy. The specimens of the tumor and matched, macroscopically unchanged renal tissue were obtained from surgically resected kidney. Specimens for RNA or protein extraction were immediately frozen in liquid nitrogen and stored at $-80^{\circ} \mathrm{C}$ until further analysis. Tumor and kidney fragments for histological and immunohistochemical studies were fixed in $4 \%$ buffered formaldehyde for $48-72 \mathrm{~h}$ at room temperature, dehydrated using a series of alcohol solutions in ascending concentrations (50,60, 70, 85 and 99.8\%; at room temperature), cleared with xylene (1:1 xylene and $99.8 \%$ alcohol solution followed by three xylene immersions; at room temperature) and processed into paraffin blocks. Clinical staging was based on the American Joint Committee on Cancer criteria (17). The tumor nuclear grading was characterized by a pathologist according to the Fuhrman system (18). Clinicopathological and demographic data of the patients as well as their overall survival (OS) records were collected during the study. The median follow-up time was 40.6 months.

RNA extraction and reverse transcription-quantitative polymerase chain reaction ( $R T-q P C R)$. Total RNA was extracted and reverse transcribed using the method described previously (19). The $I K B K B$ transcripts in tissue homogenates were determined by qPCR and normalized to peptidylprolylisomerase A (PPIA) and TATA box binding protein (TBP) mRNAs using TaqMan Fast Advanced Master Mix (Applied Biosystems; Thermo Fisher Scientific, Inc., Waltham, MA, USA) and the respective TaqMan Gene Expression Assay (IKBKB, \#Hs00233287_m1;
PPIA,\#Hs99999904_m1; and TBP,\#Hs00427620_m1; Applied Biosystems; Thermo Fisher Scientific, Inc.). qPCR reactions were performed using ABI 7500/7500 Fast Real-Time PCR System (Applied Biosystems; Thermo Fisher Scientific, Inc.) according to the protocol described by Kowalczyk et al (20). The following thermocycling conditions were used: polymerase activation for $20 \mathrm{sec}$ at $95^{\circ} \mathrm{C}$, then 40 cycles of denaturation for $3 \mathrm{sec}$ at $95^{\circ} \mathrm{C}$ and annealing/extension for $30 \mathrm{sec}$ at $60^{\circ} \mathrm{C}$. The $\Delta \Delta \mathrm{Cq}$ method (21) was used to determine the fold differences [relative quantification (RQ)] in expression between the paired samples of ccRCC and unchanged renal tissue. On the basis of $I K B K B$ RQ (ccRCC vs. renal tissue), specimens were divided into two groups, regarded as IKBKB 'upregulated' $(R Q \geq 1.5)$ and 'no change and downregulated' $(\mathrm{RQ}<1.5)$.

Protein extraction, SDS-PAGE and western blot analysis. Procedures were performed according to the method described previously (22) with some modifications. Briefly, the samples were homogenized in radioimmunoprecipitation lysis buffer supplemented with 1:100 protease inhibitor cocktail, 1:100 phosphatase inhibitor cocktail and $5 \mathrm{mM}$ EDTA (Sigma-Aldrich; Merck, KGaA, Darmstadt, Germany). Homogenates were centrifuged twice at $9,000 \mathrm{xg}$ for $10 \mathrm{~min}$ at $4^{\circ} \mathrm{C}$. The protein content in the supernatant was determined by the Bradford method. Protein lysates were denatured for $5 \mathrm{~min}$ at $95^{\circ} \mathrm{C}$ and loaded on a $10 \%$ polyacrylamide gel (30 $\mu \mathrm{g} / \mathrm{lane})$, separated (10 mA/gel during migration in stacking gel, then $15 \mathrm{~mA} / \mathrm{gel})$, transferred onto polyvinylidene difluoride membrane (Roche Diagnostics $\mathrm{GmbH}$, Mannheim, Germany) and blocked in 5\% nonfat dry milk for $2 \mathrm{~h}$ at room temperature. The level of protein in homogenates of paired tumor and renal tissue specimens was determined using rabbit anti-human antibodies against IKBKB (1:1,000; cat. no. sc-7329; Santa Cruz Biotechnology, Inc., Dallas, TX, USA) and actin (ACTB; 1:100; cat. no. A2066; Sigma-Aldrich; Merck, KGaA) as the internal loading control. Following overnight incubation at $4^{\circ} \mathrm{C}$ with primary antibodies, the membranes were treated with polyclonal horseradish peroxidase-conjugated goat anti-rabbit IgG secondary antibodies (diluted 1:40,000; cat. no. A0545; Sigma-Aldrich; Merck, KGaA) for $60 \mathrm{~min}$ at room temperature, developed with SuperSignal West Pico Chemiluminescent Substrate (Thermo Fisher Scientific, Inc.) and visualized with G:BOX iChemi XR imaging system (Syngene Europe, Cambridge, UK). Band intensity was quantified using Image J software (version 1.50i; National Institutes of Health, Bethesda, MD, USA). IKBKB/ACTB optical density (OD) ratios were used to determine fold differences in expression between the paired samples of ccRCC and unchanged renal tissue. On the basis of their relative IKBKB OD ratios (ccRCC vs. renal tissue) specimens were divided into two groups, regarded as IKBKB 'upregulated' (OD ratios $\geq 1.5$ ) and 'no change and downregulated' (OD ratios <1.5)

Immunohistochemistry (IHC) and evaluation of immunoreactivity. IKBKB immunostaining of the tumor and non-cancerous kidney $4-\mu$ m-thick paraffin sections was performed using the Autostainer Link 48 supplied with EnVision FLEX reagents (Dako; Agilent Technologies, Inc., Santa Clara, CA, USA) according to the previously described method (23). Rabbit antibody directed against human IKBKB (1:1,000; cat. no. sc-7329; Santa Cruz Biotechnology, Inc.) was applied for $20 \mathrm{~min}$ at room 

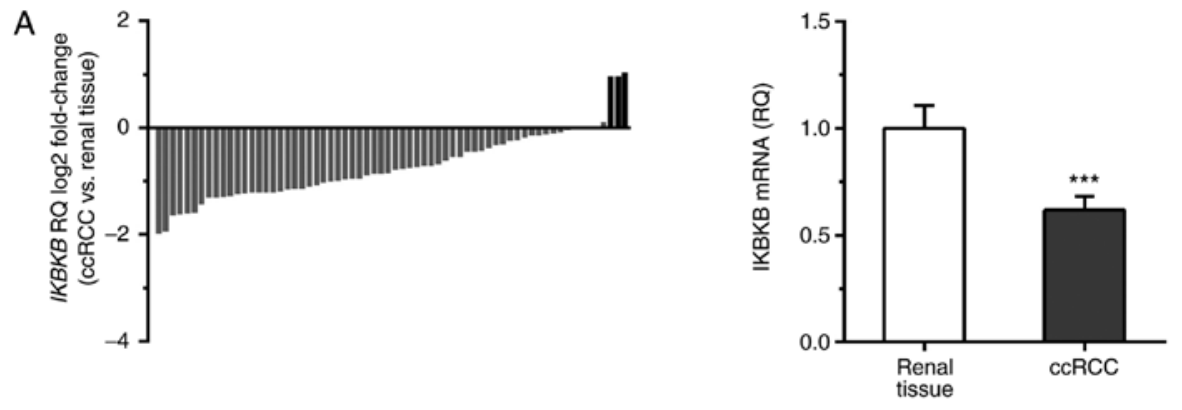

R C R C R C R C R C R C
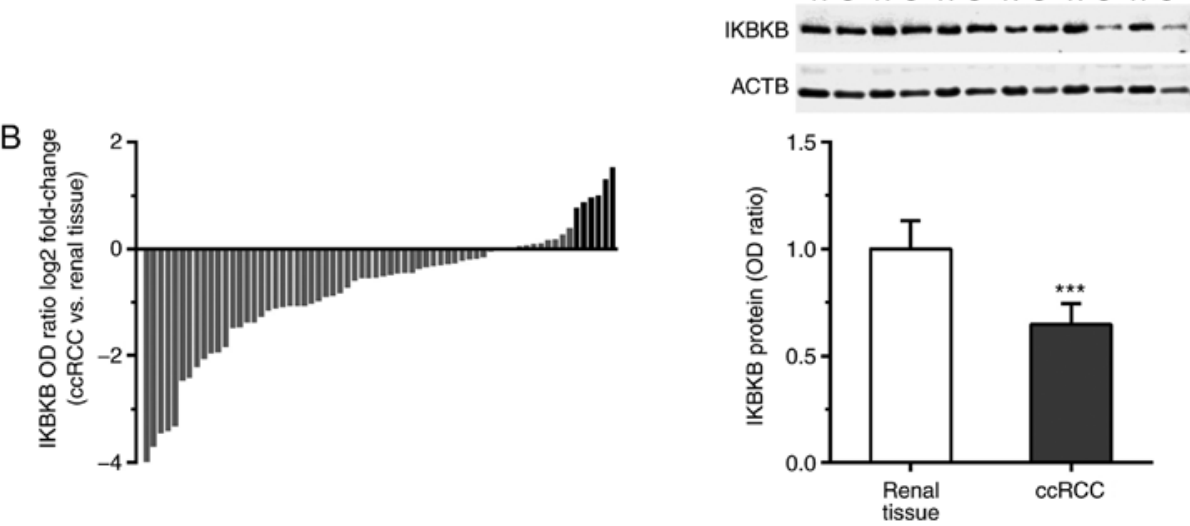

Figure 1. IKBKB expression in the ccRCC specimens and matched unchanged renal tissues at the mRNA and protein levels were determined by (A) reverse transcription-quantitative polymerase chain reaction and (B) western blot. Grey bars represent patients revealing not changed or downregulated $I K B K B$, black bars represents patients with upregulated IKBKB (left). Average IKBKB expression levels in the ccRCC specimens and unchanged renal tissues at the mRNA and protein levels (right). ${ }^{* * *} \mathrm{P}<0.001$. ccRCC, clear cell renal cell carcinoma; IKBKB, inhibitor of nuclear factor $\kappa \mathrm{B}$ kinase subunit $\mathrm{B}$.

temperature whereas the negative controls were performed by omitting the primary antibody. The sections were counterstained with EnVision FLEX Hematoxylin (ready-to-use solution; Dako; Agilent Technologies, Inc.) for $5 \mathrm{~min}$ at room temperature. The IKBKB immunoreactivity was evaluated using an Olympus BX53 light microscope (Olympus Corporation, Tokyo, Japan) by two independent pathologists in a blinded manner regarding the clinicopathological data of the patients. In doubtful cases, re-evaluation was performed until a consensus was achieved. Immunoexpression of IKBKB was assessed in the cytoplasm of cancer cells and non-transformed, normal epithelial cells of the proximal convoluted tubules (PCTs). IKBKB immunoreactivity was evaluated according to the imunoreactive score (IRS) of Remmele and Stegner (24). The IRS scale is based on the percentage of cells exhibiting positive reaction ( 0 points, absence of cells with positive reaction; 1 point, $1-10 \% ; 2$ points, $11-50 \%$; 3 points, $51-80 \%$; 4 points, $>80 \%$ cells with positive reaction) and reaction intensity ( 0 , no reaction; 1 , low intensity reaction; 2 , moderate intensity reaction; 3 , intense reaction). The final score is the result of multiplication of both parameters and ranges from 0 to 12 points). On the basis of their relative IRS ratios specimens were divided into two groups regarded as 'upregulated' (IRS ratio $\geq 1.5$ and tumor-only positive specimens) and 'no change or downregulated' IKBKB immunoreactivity (IRS ratio $<1.5$ or renal-only positive specimens).

Cancer genomics data from The Cancer Genome Atlas (TCGA). A dataset containing results of RNA-Seq analysis of 469 primary nephrectomy specimens from patients with histologically confirmed ccRCC (2) was investigated for mutations, putative copy-number alterations and expression levels of $I K B K B$ using a cBio Cancer Genomics Portal (25). The $I K B K B$ expression data were queried using 1.5 -fold change as a z-score threshold value.

Statistical analysis. Statistical analysis was performed using Prism 6.04 (GraphPad Software, Inc., La Jolla, CA, USA) and Statistica 13.1 (Statsoft, Tulsa, OK, USA). The IKBKB expression levels are expressed as mean \pm standard error. The differences in IKBKB expression levels between the paired tumor and unchanged renal tissue specimens were examined by the Wilcoxon matched-pairs test. Fisher's exact, Mann-Whitney U test and Spearman's rank correlation were used to assess associations between the patient data and $I K B K B$ expression levels. Survival curves were plotted according to the Kaplan-Meier method and the significance of differences in OS between groups of patients was evaluated by log-rank test. The uni- and multivariate survival associations were analyzed using the Cox proportional hazards regression model. $\mathrm{P}<0.05$ was considered to indicate statistically significant difference.

\section{Results}

IKBKB expression is downregulated in ccRCC at the mRNA and protein levels. All tumor and matched unchanged renal tissue samples of ccRCC patients expressed $I K B K B$ mRNA. $I K B K B$ mRNA level was reduced or remained unchanged in $63 / 66(95.5 \%)$, and was elevated in 3/66 (4.5\%) ccRCC cases (Fig. 1A). The average expression level of $I K B K B$ transcript was significantly decreased in ccRCC compared with the 
A

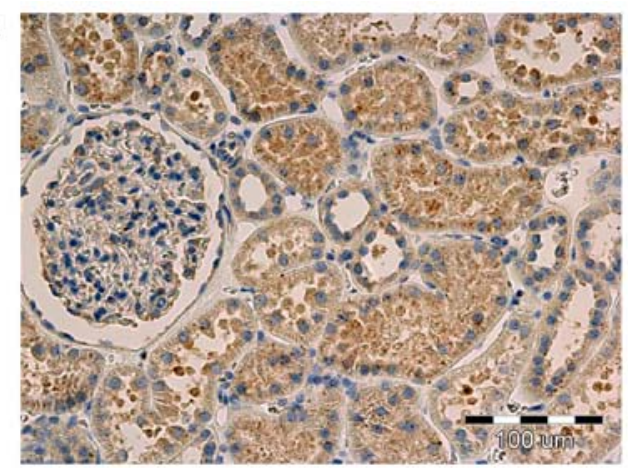

C

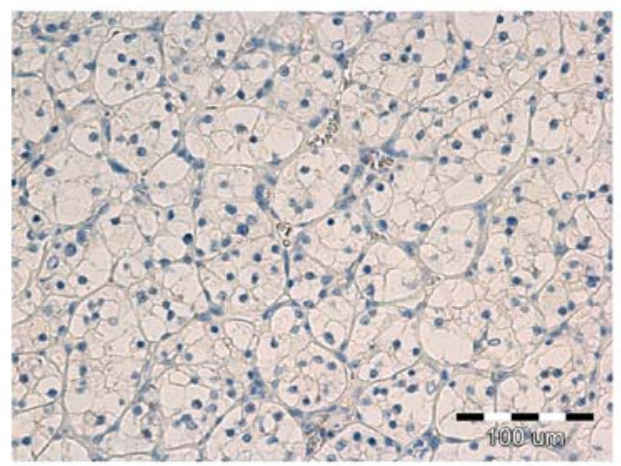

B

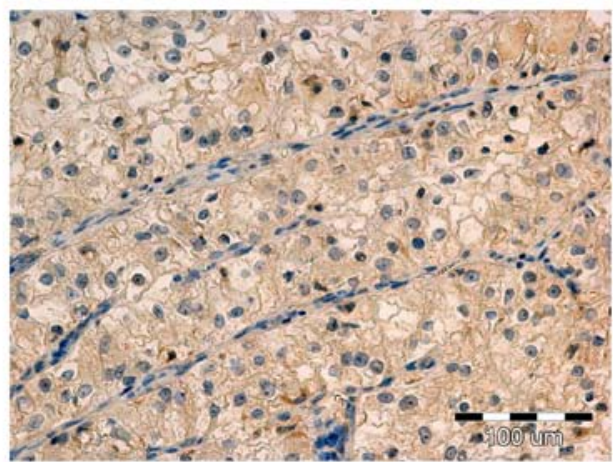

D

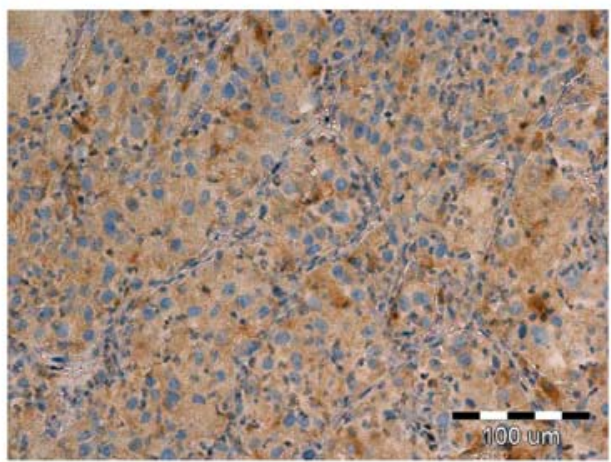

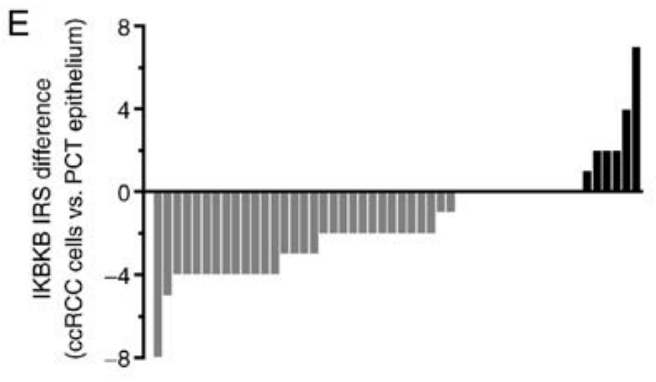

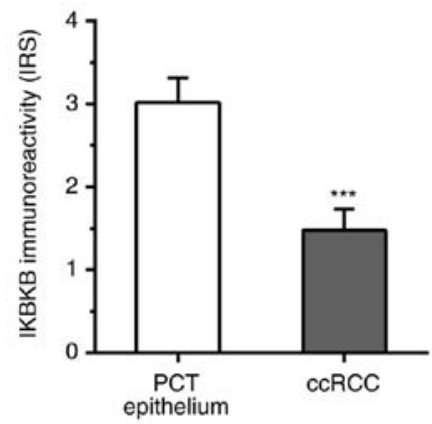

Figure 2. Evaluation of IKBKB expression in the sections of (A) unchanged kidney and (B) tumor of representative patient with ccRCC by immunohistochemistry. IKBKB protein was present in the cytoplasm but not in the nuclei of cancer and renal cells. IKBKB immunoexpression levels were heterogeneous among the analyzed tumors. (C) Many ccRCC specimens were negative for IKBKB immunoreactivity, (D) while some were characterized by moderate or strong immunoexpression of IKBKB. Original magnification, x200. (E) IKBKB IRS in ccRCC specimens and matched unchanged renal tissues. Grey bars represent patients revealing not changed or downregulated $I K B K B$, black bars represents patients with upregulated $I K B K B$ (left). Average $I K B K B$ expression levels in the ccRCC specimens and unchanged renal tissues at the mRNA and protein levels (right). ${ }^{* * *} \mathrm{P}<0.001$. IKBKB, inhibitor of nuclear factor $\kappa B$ kinase subunit B; IRS, immunoreactivity score; ccRCC, clear cell renal cell carcinoma; PCT, proximal convoluted tubules.

corresponding renal tissues (RQ $0.62 \pm 0.06$ and $1.00 \pm 0.11$ respectively; P<0.001; Fig. 1A).

IKBKB protein was present in all tested homogenates of tumor and renal tissue as determined by western blotting. The content of IKBKB protein was reduced or remained unchanged in 60/66 (90.9\%) ccRCC cases; whereas, it was elevated in $6 / 66(9.1 \%)$ of tumor homogenates (Fig. 1B). The average IKBKB/ACTB OD was significantly lower in ccRCC samples compared with the corresponding unchanged kidney tissue (OD ratio $0.65 \pm 0.10$ and $1.00 \pm 0.13$, respectively; $\mathrm{P}<0.001$; Fig. $1 \mathrm{~B}$ ).

Immunoreactivity of IKBKB was observed in the cytoplasm of cancer cells and PCT epithelial cells of analyzed sections (Fig. 2A-D). In addition to PCT epithelium, the cells of distal convoluted tubules, renal glomeruli and Bowman's capsule from non-cancerous renal tissue sections exhibited weak to moderate cytoplasmic IKBKB immunoreactivity. IKBKB did not exhibit nuclear expression in the tumor or non-cancerous renal tissue. IKBKB immunoreactivity was detected in the cancer cells of 26/50 (52\%) ccRCC cases. In the PCT epithelium of matched renal tissue, IKBKB expression was observed in 41/50 (82\%) cases. IKBKB protein expression was downregulated in 31/50 (62\%), remained unchanged in $13 / 50(26 \%)$ and was elevated in 6/50 (13\%) of ccRCC cases compared with the matched non-cancerous tissue (Fig. 2E). The average IKBKB immunoreactivity was significantly reduced in the tumor cells compared with the PCT epithelial cells of corresponding non-cancerous renal tissues (IRS $1.48 \pm 0.25$ and $3.02 \pm 0.29$, respectively; $\mathrm{P}<0.001$; Fig. $2 \mathrm{E}$ ).

Increased IKBKB immunoreactivity in ccRCC cells is associated with higher nuclear grade. Elevated immunoexpression of IKBKB protein in cancer cells compared with normal PCT epithelium was positively associated with Fuhrman nuclear grade ( $\mathrm{P}=0.0331$; Fisher's exact test; Table I). Furthermore, there was $\sim 2$-fold increase in relative immunoreactivity of IKBKB in cancer cells of G3 tumors compared with G1 and 


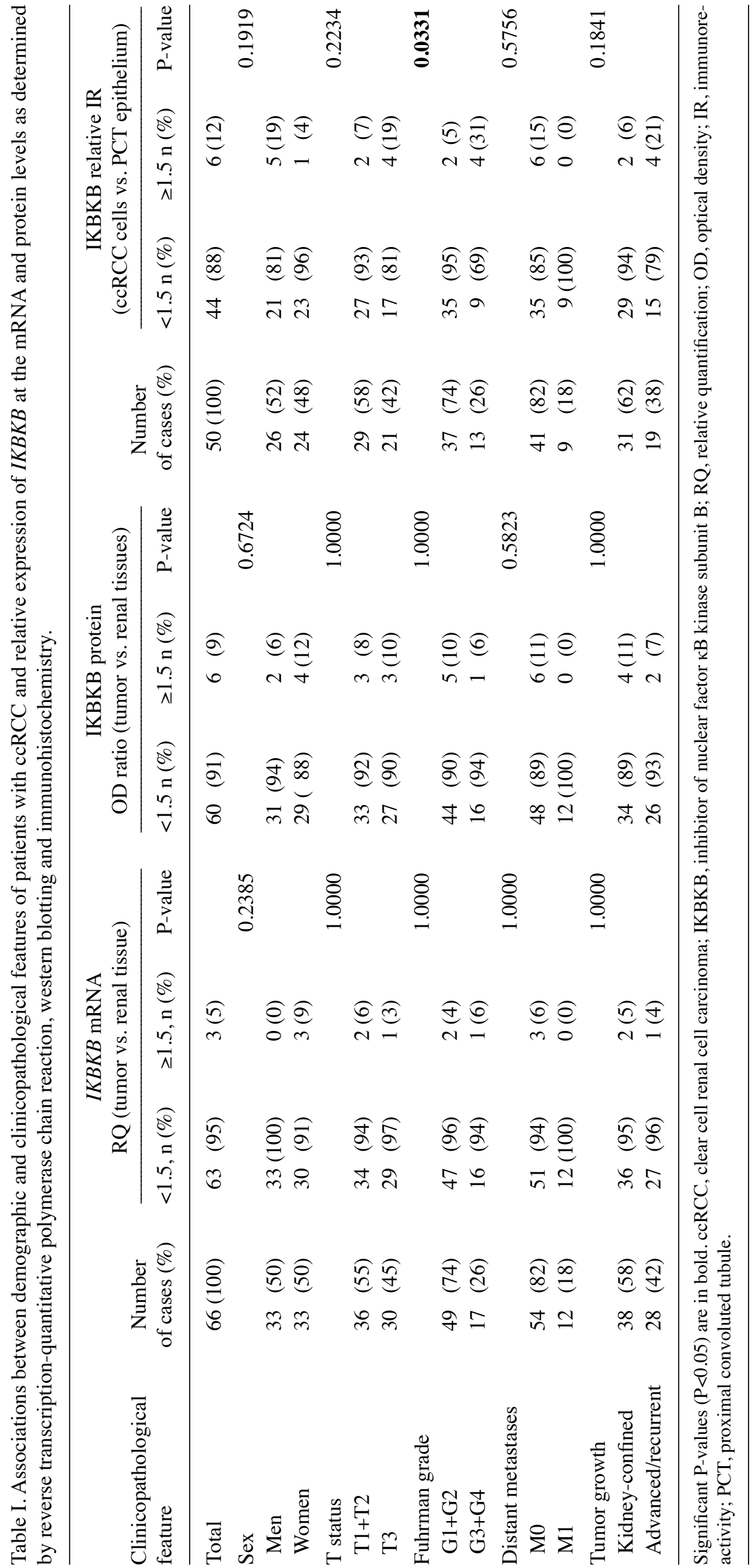


Table II. Correlation between demographic and clinicopathological features of patients with ccRCC and relative expression of $I K B K B$ at the mRNA and protein levels as determined by reverse transcription-quantitative polymerase chain reaction, western blotting and immunohistochemistry.

\begin{tabular}{|c|c|c|c|c|c|c|}
\hline \multirow[b]{2}{*}{ Variable } & \multicolumn{2}{|c|}{$\begin{array}{c}I K B K B \text { mRNA RQ } \\
\text { (tumor vs. renal tissue, } \mathrm{n}=66 \text { ) }\end{array}$} & \multicolumn{2}{|c|}{$\begin{array}{c}\text { IKBKB protein OD ratio } \\
\text { (tumor vs. renal tissues, } \mathrm{n}=66 \text { ) }\end{array}$} & \multicolumn{2}{|c|}{$\begin{array}{c}\text { IKBKB } \\
\text { relative IR (ccRCC cells } \\
\text { vs. PCT epithelium, } \mathrm{n}=50)\end{array}$} \\
\hline & Spearman's @ & P-value & Spearman's @ & P-value & Spearman's @ & P-value \\
\hline Age & -0.2291 & 0.0643 & 0.1107 & 0.3764 & -0.0983 & 0.4971 \\
\hline Tumor size & -0.0366 & 0.7704 & 0.0402 & 0.7486 & -0.0235 & 0.8713 \\
\hline
\end{tabular}

ccRCC, clear cell renal cell carcinoma; IKBKB, inhibitor of nuclear factor $\kappa \mathrm{B}$ kinase
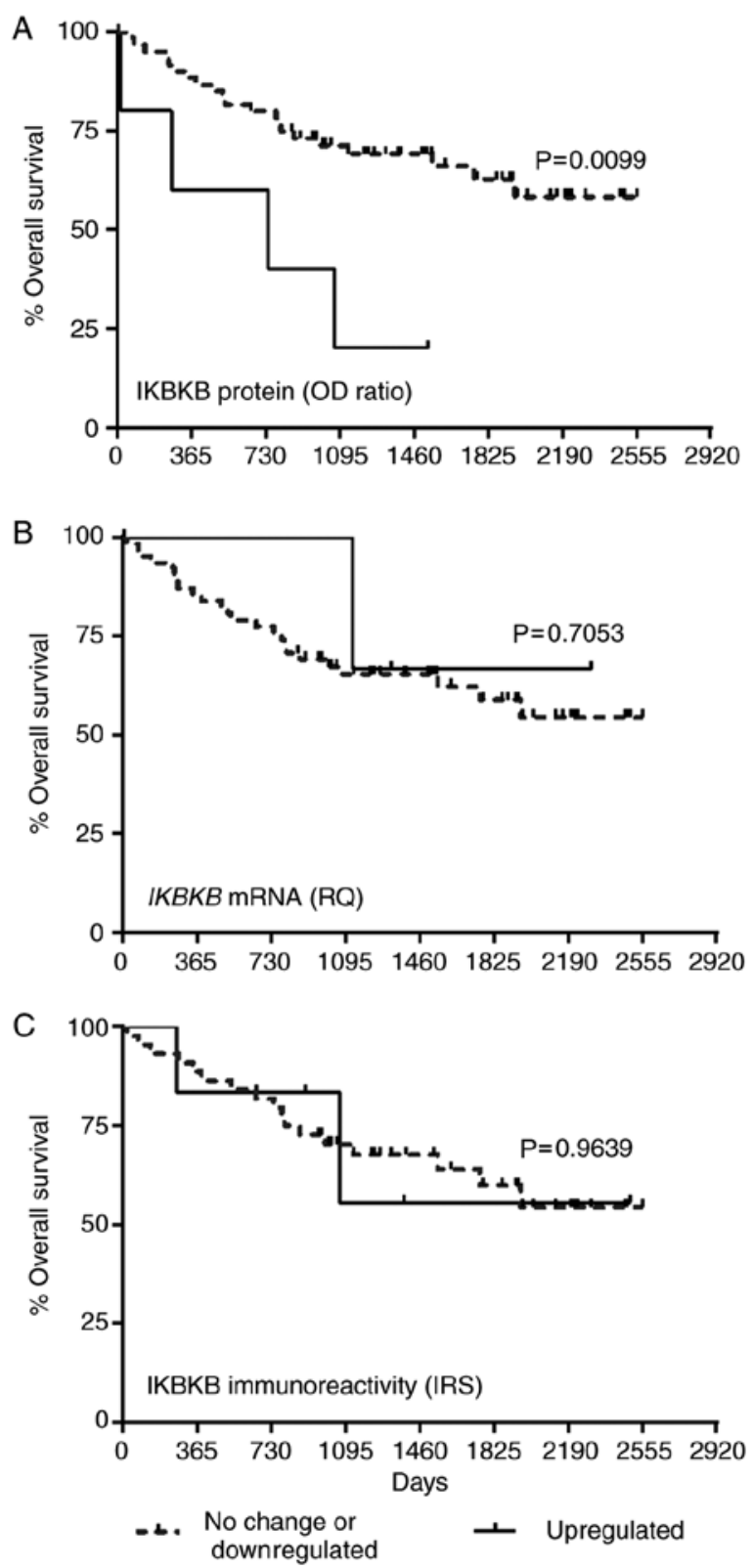

Figure 3. Kaplan-Meier diagrams of overall survival of patients with clear cell renal cell carcinoma regarding the expression levels of (A) IKBKB protein OD, (B) IKBKB mRNA and (C) IKBKB immunoreactivity. P-values for the corresponding log-rank test are shown. IKBKB, inhibitor of nuclear factor $\kappa \mathrm{B}$ kinase subunit $\mathrm{B} ; \mathrm{RQ}$, relative quantification; $\mathrm{OD}$, optical density; IRS, immunoreactivity score.
G2 tumors ( $0.92 \pm 0.17$ vs. $0.54 \pm 0.08 ; \mathrm{P}=0.0304$; Mann-Whitney $\mathrm{U}$ test). No other correlations between $I K B K B$ expression levels and demographic or clinicopathological parameters of the patients were identified (Tables I and II).

Upregulated IKBKB protein expression is associated with unfavorable prognosis. Kaplan-Meier plots presenting the OS of patients with ccRCC grouped according to the expression levels of IKBKB are presented in the Fig. 3. Increased of IKBKB protein expression was significantly associated with shorter OS of patients with ccRCC (median 25.7 months for upregulated group vs. $\geq 85.3$ months for not changed or downregulated IKBKB protein content; $\mathrm{P}=0.0099$; Fig. 3A). The expression of IKBKB transcript and immunoreactivity of IKBKB in cancer cells did not correlate with OS of patients with ccRCC (Fig. 3B and $\mathrm{C}$, respectively). Univariate Cox proportional hazards regression revealed that IKBKB protein level, T-status of the primary tumor, nuclear grade, presence of distant metastases and advanced/recurrent disease were associated with OS of the patients (Table III). The subsequent multivariate analysis confirmed that higher IKBKB protein level [hazard ratio $(\mathrm{HR})=5.50 ; \mathrm{P}=0.0020$; Table III] and with the presence of distant metastases $(\mathrm{HR}=3.99 ; \mathrm{P}=0.0182$; Table III) achieved a status of independent prognostic factors in ccRCC.

TCGA dataset analysis. Analysis of cancer genomics data provided by TCGA revealed that none of 469 ccRCC cases included in the dataset contained $I K B K B$ sequence mutation. Copy number alterations were reported only in four ccRCC cases $(0.9 \%)$. The expression level of $I K B K B$ mRNA was downregulated in 31/469 (6.6\%) and upregulated in $28(6 \%)$ of queried ccRCC samples while the levels of IKBKB protein in the tumor did not differ from those in the matching renal tissue.

\section{Discussion}

In the present study, three independent techniques (RT-qPCR, western blot analysis and IHC) were used to demonstrate that the expression level of $I K B K B$ is decreased in ccRCC. The majority of the analyzed tumor specimens exhibited reduced levels of $I K B K B$ mRNA and protein. However, an upregulated IKBKB protein level was associated with higher nuclear grade of the tumor and significantly shorter survival, suggesting an oncogenic role of this kinase in ccRCC. 
Table III. Univariate and multivariate Cox regression analysis of the overall survival rates associated with different prognostic variables in patients with clear cell renal cell carcinoma.

\begin{tabular}{|c|c|c|c|c|c|c|}
\hline \multirow[b]{2}{*}{ Parameter } & \multicolumn{3}{|c|}{ Univariate Cox regression } & \multicolumn{3}{|c|}{$\begin{array}{l}\text { Multivariate } \\
\text { Cox regression }\end{array}$} \\
\hline & HR & $95 \% \mathrm{CI}$ & P-value & HR & $95 \% \mathrm{CI}$ & P-value \\
\hline$I K B K B$ mRNA RQ (upregulated vs. no change/downregulated) & 0.47 & $0.06-3.50$ & 0.4645 & & & \\
\hline IKBKB protein OD ratio (upregulated vs. no change/downregulated) & 4.61 & $1.70-12.5$ & 0.0026 & 5.50 & $1.86-16.2$ & 0.0020 \\
\hline IKBKB immunoreactivity (upregulated vs. no change/downregulated) & 1.03 & $0.24-4.49$ & 0.9634 & & & \\
\hline Sex (women vs. men) & 0.70 & $0.32-1.51$ & 0.3604 & & & \\
\hline Age (years) & 0.99 & $0.95-1.03$ & 0.5269 & & & \\
\hline Tumor size (cm) & 1.17 & $1.05-1.31$ & 0.0052 & 1.07 & $0.88-1.28$ & 0.5094 \\
\hline Depth of invasion (T3 vs. T1+T2) & 4.31 & $1.80-10.3$ & 0.0010 & 3.12 & $0.87-11.2$ & 0.0815 \\
\hline Fuhrman grade (G3 vs. G1+G2) & 3.33 & $1.49-7.44$ & 0.0034 & 1.65 & $0.63-4.33$ & 0.3112 \\
\hline Distant metastases (M1 vs. M0) & 2.78 & $1.19-6.50$ & 0.0185 & 3.99 & $1.27-12.6$ & 0.0182 \\
\hline Tumor growth (advanced/recurrent vs. kidney-confined) & 2.37 & $1.08-5.20$ & 0.0318 & 0.58 & $0.19-1.77$ & 0.3412 \\
\hline
\end{tabular}

Median follow-up time, 40.6 months. Significant $\mathrm{P}$-values $(\mathrm{P}<0.05)$ are in bold. HR, hazard ratio; CI, confidence interval; RQ, relative quantification; OD, optical density.

Previous studies using patients with RCC, cell lines and xenograft animal models of RCC identified $I K B K B$ as a factor associated with the progression of the disease and poor patient outcomes. A dataset from TCGA containing 469 ccRCC cases revealed that copy number alterations of $I K B K B$ were rare in this cancer ( $1 \%$ of tumors), and no alterations in the $I K B K B$ sequence were reported in this dataset $(2,25)$. NF- $\kappa B$ pathway genes rarely contain mutations in cancer (12). Constitutive activation of $\mathrm{NF}-\kappa \mathrm{B}$ in certain cancers is usually mediated by alterations of upstream regulators and/or altered expression levels of NF- $\kappa \mathrm{B}$-associated genes (12). In the current study, $I K B K B$ expression was also investigated using the TCGA data provided by the cBio Cancer Genomics Portal $(2,25)$ setting a z-score threshold for 1.5 -fold change. This evaluation revealed that the expression level of $I K B K B$ transcript determined by RNA-Seq was downregulated in $7 \%$ and upregulated in $6 \%$ of ccRCC cases, while IKBKB protein levels measured by reverse-phase protein array remained unaltered in all 469 samples included in the study $(2,25)$. Although these expression data are not in line with the results of the current study, this may be attributed to different sensitivities and specificities of methods used. However, the prognostic significance of IKBKB reported in this study is in accordance with analyses based on previously published study by Peri et al (26). Meta-analysis of ccRCC expression datasets identified a subset of genes including $I K B K B$ and NF- $\mathrm{NB}$ mediators, matrix metalloproteinase 9 , proteasome subunit $\beta 9$ and superoxide dismutase 2, whose elevated expression levels were associated with worse patient outcomes (26). Another study established a model linking pVHL loss to increased activation of NF- $\kappa \mathrm{B}$, potentially elucidating a previously reported observation that loss of may be associated with the chemoresistance of ccRCC to tumor necrosis factor therapy (27). Recently, it has been reported that IKBKB protein may indirectly have a role in stabilizing HIF1 $\alpha$ and HIF2 $\alpha$ in RCC cells via activation of NF- $\mathrm{NB}$ essential modulator (NEMO) protein, a regulatory subunit of the IKK complex (28). In addition, it was demonstrated that the degree of NEMO protein expression was downregulated in $62.8 \%$ tumor samples derived from 250 patients with ccRCC, and it was positively associated with the progression of ccRCC (28). This observation seems to be analogous to the finding that IKBKB immunoreactivity was decreased in the majority of analyzed ccRCC specimens, while increased IKBKB protein expression was associated with higher tumor grade and poorer patient outcomes. However, additional studies are required to investigate unknown links between the IKBKB and NEMO proteins and potential common mechanisms underlying their altered expression in ccRCC.

Beneficial effects of decreased $I K B K B$ expression and/or IKBKB protein activity on the effectiveness of different anticancer approaches were reported previously (29). Treatment of metastatic RCC cell lines, ACHN and SN12K1, with an anti-oxidant agent, pyrrolidine dithiocarbamate, decreased expression level of IKBKB and other components of $\mathrm{NF}-\kappa \mathrm{B}$ signaling, as assessed by western blotting (30). The resulting inhibition of $\mathrm{NF}-\kappa \mathrm{B}$ pathways exerted anti-proliferative, pro-apoptotic and anti-angiogenic effects in the tested cell lines $(30,31)$ and sensitized them to cisplatin treatment (32). Further experiments performed in a xenograft animal model of RCC demonstrated that the antioxidant treatment reduced cancer cell proliferation and attenuated tumor progression, with decreased expression of $\mathrm{NF}-\kappa \mathrm{B}$ proteins and upstream kinases, IKBKB and IKKA (33). Notably, accumulation of reactive oxygen species in the kidneys of superoxide-deficient mice resulted in decreased expression of IKBKB (34), suggesting that the influence of oxidative stress on expression of NF- $\kappa \mathrm{B}$-associated genes is tissue- or disease-specific. Another study performed in RCC cell lines demonstrated that IKBKB and RelA (p65) were required for the oncogenic properties of microRNA-21 (miR-21) in ACHN cells (35). In turn, miR-21 indirectly increased IKBKB phosphorylation, which upregulated NF- $\mathrm{B}$ and mechanistic target of rapamycin complex 1 signaling, resulting in enhanced proliferation, migration and invasiveness of ACHN and 786-O 
cell lines $(35,36)$. Transfection of RCC cell lines with a plasmid expressing IKBKB attenuated sunitinib-induced p53 promoter transcriptional activity (37). These reports suggest that increased $I K B K B$ content in cancer cells can compromise the effectiveness of anticancer therapy, suggesting potential usefulness of $I K B K B$ as both prognostic and predictive marker in ccRCC.

Altered IKBKB expression and/or IKBKB kinase activity were previously reported to be associated the with occurrence and progression of several types of human cancer, including breast cancer, pancreatic cancer, thyroidal C-cells carcinoma, acute myeloid leukemia and ovarian cancer $(7,8,12,29,38)$. However, the available data concerning IKBKB expression are inconclusive. $I K B K B$ transcript levels were significantly upregulated in human hepatocellular carcinoma compared with adjacent normal tissue (39). Cultured Hs578T breast cancer cells exhibited aberrant expression and activity of IKBKB compared with untransformed mammary epithelial cells (40). Reduced $I K B K B$ expression were demonstrated in glioblastoma tissues at the mRNA and protein levels (41). However, downregulation of $I K B K B$ was attributed to microglia/macrophages infiltrating advanced glioblastoma tumors, indicating the important role of this kinase for the local microenvironment and antitumor response (41).

In conclusion, to the best of our knowledge, the present study is the first comprehensive investigation analyzing $I K B K B$ expression at the mRNA and protein levels in a cohort of patients with ccRCC. The results suggest that the three techniques applied to determine the IKBKB expression, RT-qPCR, western blotting and IHC, should be used as a complementary rather than alternative methods. However, additional methodological studies are required to compare these and other available assays before conclusions are made. The number of patients included in the study was enough to disclose associations of IKBKB protein expression level with clinicopathological parameters and survival of patients with ccRCC. Therefore, the findings of the present study demonstrate that the expression of IKBKB protein may be of clinical relevance in ccRCC. The elevated content of IKBKB in the ccRCC tumor tissue may be useful as a potential marker of prognosis, and suggests that the application of anti-NF- $\mathrm{kB}$ treatments may sensitize ccRCC cells to certain adjuvant therapies. Further studies using a larger number of patients are required to support this suggestion, and validate prognostic or predictive value of IKBKB in ccRCC.

\section{Acknowledgements}

The authors wish to thank Dr Aleksandra Piotrowska from the Department of Human Morphology and Embryology, Division of Histology and Embryology, Wroclaw Medical University (Wroclaw, Poland) for technical support.

\section{Funding}

This study was supported by the National Science Centre (Poland; grant no. 2012/05/B/NZ4/01832).

\section{Availability of data and materials}

The datasets used and/or analyzed during the current study are available from the corresponding author on reasonable request.

\section{Authors' contributions}

BEK, JK and ZK designed the study, analyzed and interpreted the results; BEK and JK wrote the manuscript draft; ZK corrected the final version of the manuscript; JGo and PK collected clinical samples; JGo collected clinicopathological and survival data; BEK, JK, AEK, ASJ performed qPCR and western blot assays; JGr and PD performed IHC and evaluated immunoreactivity; BEK performed the statistical analysis; JK and BEK were managing the project. All authors read and approved the final manuscript.

\section{Ethics approval and consent to participate}

This study was approved by the Bioethics Committee for Scientific Research at the University of Warmia and Mazury in Olsztyn (Olsztyn, Poland; agreements no. 4/2010 and 44/2011). Written informed consent (as specified in the Declaration of Helsinki) was obtained from each patients included in the study.

\section{Patient consent for publication}

Not applicable.

\section{Competing interests}

The authors declare that they have no competing interests.

\section{References}

1. Greef B and Eisen T: Medical treatment of renal cancer: New horizons. Br J Cancer 115: 505-516, 2016.

2. Cancer Genome Atlas Research Network: Comprehensive molecular characterization of clear cell renal cell carcinoma. Nature 499: 43-49, 2013.

3. Znaor A, Lortet-Tieulent J, Laversanne M, Jemal A and Bray F: International variations and trends in renal cell carcinoma incidence and mortality. Eur Urol 67: 519-530, 2015.

4. Shoji S, Nakano M, Sato H, Tang XY, Osamura YR, Terachi T, Uchida T and Takeya K: The current status of tailor-made medicine with molecular biomarkers for patients with clear cell renal cell carcinoma. Clin Exp Metastasis 31: 111-134, 2014.

5. Bhatt JR and Finelli A: Landmarks in the diagnosis and treatment of renal cell carcinoma. Nat Rev Urol 11: 517-525, 2014.

6. DiDonato JA, Mercurio F and Karin M: NF- $\mathrm{BB}$ and the link between inflammation and cancer. Immunol Rev 246: 379-400, 2012.

7. Bradford JW and Baldwin AS: IKK/nuclear factor-kappaB and oncogenesis: Roles in tumor-initiating cells and in the tumor microenvironment. Adv Cancer Res 121: 125-145, 2014.

8. Morais C, Gobe G, Johnson DW and Healy H: The emerging role of nuclear factor kappa B in renal cell carcinoma. Int J Biochem Cell Biol 43: 1537-1549, 2011.

9. DiDonato JA, Hayakawa M, Rothwarf DM, Zandi E and Karin M: A cytokine-responsive IkappaB kinase that activates the transcription factor NF-kappaB. Nature 388: 548-554, 1997.

10. Li ZW, Chu W, Hu Y, Delhase M, Deerinck T, Ellisman M, Johnson R and Karin M: The IKKbeta subunit of IkappaB kinase (IKK) is essential for nuclear factor kappaB activation and prevention of apoptosis. J Exp Med 189: 1839-1845, 1999.

11. Tanaka M, Fuentes ME, Yamaguchi K, Durnin $\mathrm{MH}$, Dalrymple SA, Hardy KL and Goeddel DV: Embryonic lethality, liver degeneration, and impaired NF-kappa B activation in IKK-beta-deficient mice. Immunity 10: 421-429, 1999.

12. Erstad DJ and Cusack JC Jr: Targeting the NF- $\kappa B$ pathway in cancer therapy. Surg Oncol Clin N Am 22: 705-746, 2013.

13. Xia Y, Padre RC, De Mendoza TH, Bottero V, Tergaonkar VB and Verma IM: Phosphorylation of p53 by IkappaB kinase 2 promotes its degradation by beta-TrCP. Proc Natl Acad Sci USA 106: 2629-26234, 2009. 
14. Hu MC, Lee DF, Xia W, Golfman LS, Ou-Yang F, Yang JY, Zou Y, Bao S, Hanada N, Saso H, et al: IkappaB kinase promotes tumorigenesis through inhibition of forkhead FOXO3a. Cell 117: 225-237, 2004

15. Cummins EP, Berra E, Comerford KM, Ginouves A, Fitzgerald KT, Seeballuck F, Godson C, Nielsen JE, Moynagh P, Pouyssegur J and Taylor CT: Prolyl hydroxylase-1 negatively regulates IKappaB kinase-beta, giving insight into hypoxia-induced NFkappaB activity. Proc Natl Acad Sci USA 103: 18154-18159, 2006.

16. Wang Y, Zhao W, Gao Q, Fan L, Qin Y, Zhou H, Li M and Fang J: pVHL mediates K63-linked ubiquitination of IKK $\beta$, leading to IKK $\beta$ inactivation. Cancer Lett 383: 1-8, 2016.

17. Greene FL, Page DL, Fleming ID, Fritz AG, Balch CM, Haller DG and Morrow M:Kidney. In: American Joint Committee on Cancer: AJCC Cancer Staging Manual. 6th edition. Springer, New York, NY, pp323-328, 2002.

18. Fuhrman SA, Lasky LC and Limas C: Prognostic significance of morphologic parameters in renal cell carcinoma. Am J Surg Pathol 6: 655-663, 1982.

19. Sliwinska-Jewsiewicka A, Kowalczyk AE, Krazinski BE, Godlewski J, Kwiatkowski P, Kiewisz J, Grzegrzolka J, Dziegiel P and Kmiec Z: Decreased expression of SATB2 associates with tumor growth and predicts worse outcome in patients with clear cell renal cell carcinoma. Anticancer Res 38: 839-846, 2018.

20. Kowalczyk AE, Krazinski BE, Godlewski J, Grzegrzolka J, Kiewisz J, Kwiatkowski P, Sliwinska-Jewsiewicka A, Dziegiel P and Kmiec Z: SATB1 is down-regulated in clear cell renal cell carcinoma and correlates with MIR-21-5p overexpression and poor prognosis. Cancer Genomics Proteomics 13: 209-217, 2016.

21. Livak KJ and Schmittgen TD: Analysis of relative gene expression data using real-time quantitative PCR and the 2(-Delta Delta C(T)) method. Methods 25: 402-408, 2001.

22. Kowalczyk AE, Krazinski BE, Godlewski J, Kiewisz J, Kwiatkowski P, Sliwinska-Jewsiewicka A, Kiezun J, Wierzbicki PM, Bodek G, Sulik M and Kmiec Z: Altered expression of the PLAGL1 (ZAC1/LOT1) gene in colorectal cancer: Correlations to the clinicopathological parameters. Int J Oncol 47: 951-962, 2015.

23. Kowalczyk AE, Godlewski J, Krazinski BE, Kiewisz J, Sliwinska-Jewsiewicka A, Kwiatkowski P, Pula B, Dziegiel P, Janiszewski J, Wierzbicki PM and Kmiec Z: Divergent expression patterns of SATB1 mRNA and SATB1 protein in colorectal cancer and normal tissues. Tumor Biol 36: 4441-4452, 2015.

24. Remmele W and Stegner HE: Recommendation for uniform definition of an immunoreactive score (IRS) for immunohistochemical estrogen receptor detection (ER-ICA) in breast cancer tissue. Pathologe 8: 138-140, 1987 (In German).

25. Cerami E, Gao J, Dogrusoz U, Gross BE, Sumer SO, Aksoy BA, Jacobsen A, Byrne CJ, Heuer ML, Larsson E, et al: The cBio cancer genomics portal: An open platform for exploring multidimensional cancer genomics data. Cancer Discov 2: 401-404, 2012.

26. Peri S, Devarajan K, Yang DH, Knudson AG and Balachandran S: Meta-analysis identifies NF- $\mathrm{KB}$ as a therapeutic target in renal cancer. PLoS One 8: e76746, 2013.

27. Qi $\mathrm{H}$ and Ohh M: The von Hippel-Lindau tumor suppressor protein sensitizes renal cell carcinoma cells to tumor necrosis factor-induced cytotoxicity by suppressing the nuclear factor-kappaB-dependent antiapoptotic pathway. Cancer Res 63 7076-7080, 2003.
28. Nowicka AM, Häuselmann I, Borsig L, Bolduan S, Schindler M, Schraml P, Heikenwalder M and Moch H: A novel pVHL-independent but NEMO-driven pathway in renal cancer promotes HIF stabilization. Oncogene 35: 3125-3138, 2016.

29. Gamble C, McIntosh K, Scott R, Ho KH, Plevin R and Paul A: Inhibitory kappa B kinases as targets for pharmacological regulation. Br J Pharmacol 165: 802-819, 2012.

30. Morais C, Pat B, Gobe G, Johnson DW and Healy H: Pyrrolidine dithiocarbamate exerts anti-proliferative and pro-apoptotic effects in renal cell carcinoma cell lines. Nephrol Dial Transplant 21: 3377-3388, 2006.

31. Morais C, Gobe G, Johnson DW and Healy H: Anti-angiogenic actions of pyrrolidine dithiocarbamate, a nuclear factor kappa B inhibitor. Angiogenesis 12: 365-379, 2009.

32. Morais C, Gobe G, Johnson DW and Healy H: Inhibition of nuclear factor kappa $\mathrm{B}$ transcription activity drives a synergistic effect of pyrrolidine dithiocarbamate and cisplatin for treatment of renal cell carcinoma. Apoptosis 15: 412-425, 2010.

33. Morais C, Healy H, Johnson DW and Gobe G: Inhibition of nuclear factor kappa B attenuates tumour progression in an animal model of renal cell carcinoma. Nephrol Dial Transplant 25: 1462-1474, 2010.

34. Brzóska K, Sochanowicz B, Siomek A, Olinski R and Kruszewski M: Alterations in the expression of genes related to $\mathrm{NF}-\kappa \mathrm{B}$ signaling in liver and kidney of CuZnSOD-deficient mice. Mol Cell Biochem 353: 151-157, 2011.

35. Bera A, Ghosh-Choudhury N, Dey N, Das F, Kasinath BS, Abboud HE and Choudhury GG: NFKB-mediated cyclin D1 expression by microRNA-21 influences renal cancer cell proliferation. Cell Signal 25: 2575-2586, 2013.

36. Bera A, Das F, Ghosh-Choudhury N, Kasinath BS, Abboud HE and Choudhury GG: microRNA-21-induced dissociation of PDCD4 from rictor contributes to Akt-IKK $\beta$-mTORC1 axis to regulate renal cancer cell invasion. Exp Cell Res 328: 99-117, 2014.

37. Zhu Y, Xu L, Zhang J, Hu X, Liu Y, Yin H, Lv T, Zhang H, Liu L, An H, et al: Sunitinib induces cellular senescence via p53/Dec1 activation in renal cell carcinoma cells. Cancer Sci 104: 1052-1061, 2013

38. Kim HJ, Hawke N and Baldwin AS: NF-kappaB and IKK as therapeutic targets in cancer. Cell Death Differ 13: 738-747, 2006.

39. Jiang R, Xia Y, Li J, Deng L, Zhao L, Shi J, Wang X and Sun B: High expression levels of IKKalpha and IKKbeta are necessary for the malignant properties of liver cancer. Int J Cancer 126: 1263-1274, 2010

40. Romieu-Mourez R, Landesman-Bollag E, Seldin DC, Traish AM, Mercurio F and Sonenshein GE: Roles of IKK kinases and protein kinase CK2 in activation of nuclear factor-kappaB in breast cancer. Cancer Res 61: 3810-3818, 2001

41. Mieczkowski J, Kocyk M, Nauman P, Gabrusiewicz K, Sielska M, Przanowski P, Maleszewska M, Rajan WD, Pszczolkowska D, Tykocki T, et al: Down-regulation of IKK $\beta$; expression in glioma-infiltrating microglia/macrophages is associated with defective inflammatory/immune gene responses in glioblastoma. Oncotarget 6: 33077-33090, 2015. 